THU0013 DOWNREGULATION OF MICRORNA MAY CONTRIBUTE TO ACTIVATION OF THE INTERFERON SIGNALLING PATHWAY IN THE IDIOPATHIC INFLAMMATORY MYOPATHIES

J.E. Parkes ${ }^{1}$, H. Chinoy ${ }^{2}$, P.J. Day ${ }^{3,4}$, J.A. Lamb ${ }^{1} .{ }^{1}$ Division of Population Health, Health Services Research and Primary Care; ${ }^{2}$ National Institute for Health Research Manchester Biomedical Research Centre; ${ }^{3}$ Manchester Institute of Biotechnology; ${ }^{4}$ Division of Evolution and Genomic Sciences, University of Manchester, Manchester, UK

Background: The idiopathic inflammatory myopathies (IIM) are a group of rare autoimmune conditions characterised by weakness and inflammation of skeletal muscle. MicroRNA are short single stranded RNA which regulate gene expression by binding to particular target mRNA and suppressing translation or inducing degradation. MicroRNA are known to play a role in muscle homeostasis and immune regulation and therefore may be involved in IIM pathology.

Objectives: To profile both microRNA and mRNA in whole blood samples from polymyositis (PM), dermatomyositis (DM) and inclusion body myositis (IBM) patients compared to non-myositis controls using next generation RNA sequencing.

Methods: Whole blood samples in tempus tubes were collected as part of the myositis research tissue bank held at the University of Manchester. Total RNA was extracted from 7 PM, 7 DM, 5 IBM and 5 non-myositis control samples and both microRNA and mRNA profiles were determined by sequencing on the Illumina HiSeq 4000.

Results: Analysis of microRNA expression identified 19 microRNAs as significantly differentially expressed $(D E)(p<0.05)$ for $\mathrm{PM}, 11$ for $\mathrm{DM}$ and 27 for IBM, compared to controls. Analysis of myositis specific autoantibody anti-Jo1 positive cases (four PM and one DM) vs controls identified 18 DE microRNAs, 12 of which were not identified for any of the clinical subgroups.

Gene expression analysis identified 129 significantly DE genes for PM (false discovery rate <0.05), 53 for DM, 24 for IBM and 691 for anti-Jo1 compared to controls. Ingenuity pathway analysis (IPA) of RNAseq data identified the most significant canonical pathway as 'Interferon signalling' which was predicted to be upregulated in PM, DM and anti-Jo1 subgroups. The anti-Jo1 subgroup had a higher number of DE genes and a higher activation score in the interferon signalling pathway compared to PM or DM. The IPA tool microRNA target filter was used to match DE microRNA with corresponding DE mRNA predicted or previously observed to be targets. When mRNA targets were filtered for involvement in interferon signalling, two downregulated microRNA targeted two upregulated mRNA in PM and five downregulated microRNA targeted five upregulated mRNA in the anti-Jo1 group as shown in figure 1. No microRNAs targeting mRNA involved in the interferon signalling pathway were identified for DM or IBM.

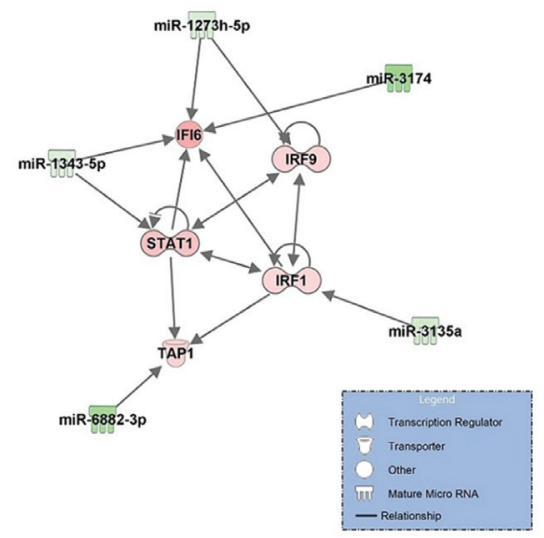

Abstract THU0013 - Figure 1. Relationship between differentially expressed microRNA in anti-Jo1 samples versus controls and mRNA targets involved in the interferon signalling pathway. Green colouring indicates downregulation; red colouring indicates upregulation and darker shades indicate greater fold change.

Conclusions: To our knowledge this is the first mRNA and microRNA profiling study in whole blood from myositis patients using RNA sequencing. Grouping by anti-Jo1 positivity identified different microRNA and increased predicted activation of interferon signalling compared to the clinical subgroups analysed. Our results suggest that increased activation of the interferon pathway in anti-Jo1 positive patients may be due to downregulation of microRNAs which target genes in this pathway.

Disclosure of Interest: None declared

DOI: 10.1136/annrheumdis-2018-eular.5593

\section{THU0014 \\ GENETIC SCREENING FOR IDENTIFYING HUMAN GENES REGULATING FIBROSIS IN SYSTEMIC SCLEROSIS}

M. Krishnan ${ }^{1}$, U. Unniyampurathu ${ }^{1}$, S. Albani ${ }^{2}$, A.H.L. Low ${ }^{3} .{ }^{1}$ Program on Emerging Infectious Diseases, Duke-National University Hospital, Singapore; ${ }^{2}$ SingHealth Translational Immunology and Inflammation Centre; ${ }^{3}$ Singapore General Hospital, Duke-National University Hospital, Singapore, Singapore, Singapore

Background: Systemic sclerosis (SSc) is a prototypic multi-systemic fibrotic disease. Fibrosis involves exacerbated collagen production. A lack of effective therapies has partly been due to incomplete understanding of the mechanisms of fibrosis and inadequate subsetting of this heterogeneous disease to allow targeted treatment. Identification of a collagen-synthesis gene signature in SSc will enhance our ability to molecularly subset SSc patients. We reasoned that there exists several unidentified genes regulating collagen synthesis, whose dysregulation play key roles in fibrosis underlying SSc.

Objectives: To discover novel genes/pathways involved in elevated collagen synthesis

Methods: We conducted a pioneering forward-genetics approach using genomewide ribonucleic acid interference-(RNAi) -screening. We performed RNAiscreening in dermal fibroblasts from three patients with early diffuse cutaneous systemic sclerosis (onset from the first non-Raynaud's manifestation of less than 3 years). Dermal fibroblasts were immortalised using ectopic expression of human telomerase. The assay used a collagen promoter driver reporter system for identifying genes regulating collagen transcription.

Results: The genetic screen identified 187 genes whose silencing altered the activation of collagen promoter activity. This included $82 \%$ and $18 \%$ positive and negative regulators, respectively. A system-level view of genes and signalling pathways regulating collagen synthesis was generated using bioinformatics analysis.

Conclusions: We anticipate that this catalogue of collagen expression regulating genes will help to unravel the dysregulated genetic regulatory modules of fibrosis and may aid in the diagnostics of different clinical subsets of SSc.

Disclosure of Interest: None declared

DOI: 10.1136/annrheumdis-2018-eular.4403

\section{THU0015 OESTROGEN DEPENDENT REGULATION OF MICRO- RNA IN RHEUMATOID ARTHRITIS}

K. Andersson ${ }^{1}$, R. Pullerits ${ }^{1}$, H. Forsblad-d'Elia ${ }^{1}, 2$, M. Erlandsson ${ }^{1}$, S

T. Silfverswärd ${ }^{1}$, M. Bokarewa ${ }^{1} .{ }^{1}$ Dept Rheumatology and inflammation research, Inst Medicine, Göteborg university, Göteborg; ${ }^{2}$ Depr Public Health and Clinical Medicine, Inst Rheumatology, Umeå University, Umeå, Sweden

Background: Oestrogen has ameliorating effects on rheumatoid arthritis (RA) Oestrogen receptor (ER) signalling affects micro-RNA ( $\mathrm{miR}$ ) expression and processing in breast cancer, while its effect on miR profile in RA have never been studied.

Objectives: To study the effect of the oestrogen replacement therapy and oestrogen receptor signalling on the miR transcription and bioprocessing in $\mathrm{RA}$ patients.

Methods: The expression of the key miR processing enzymes Dicer, Drosha and DGCR8 was measured in the leukocytes of the peripheral blood of 145 female RA patients (age 53 years, disease duration 9.8 years) by RT-PCR and analysed with respect to the levels of $E R \alpha$, used as surrogate marker of active ER $\alpha$ signalling. Total RNA was prepared from the serum samples of 46 postmenopausal female RA patients who received treatment with oestradiol (E2), noretisterone acetate, vitamin $D_{3}$ and calcium $(n=22$, mean E2 levels $229.4 \pm 143.2 \mathrm{pg} / \mathrm{ml}$ ) or vitamin $D_{3}$ and calcium supplementation only $(n=24$, mean E2 levels $30.8 \pm 8.7 \mathrm{pg} / \mathrm{ml}$ ). Serum was obtained after 12 and 24 months of treatment and was stored at $-70 \hat{E} \mathrm{C}$ until the time of analysis. MicroRNA transcription was performed by 3D-Gene microarray measuring $>2560$ miRs (TATAA Biocenter, Gothenburg).

Results: A higher $E R \alpha$ expression in RA female patients was associated with increased transcription of the major miR processing proteins Dicer $(p=0.0057)$, Drosha $(p=0.019)$ and DGRC8 $(p=0.0095)$. This activated transcription of miR biomachinery could indicate a higher demand and a facilitated miR maturation. The E2-treatment significantly changed the profile, but not the levels, of miRs in serum, where only $50 \%$ of the miR transcripts were present both in E2-treated and control samples. Also the profile of miRs related to RA and of those regulating translation of ER $\alpha$ and Dicer, Drosha, and DGCR8 proteins was affected. Extensive bioinformatic analysis of the miR-targeting genes affected during E2-treatment, revealed a reduction of gene clusters aiding autoimmune pathology and the RA-associated molecular processes including DNA replication $(p=2.6 \times 10-4)$, peptidyl-serine phosphorylation $(\mathrm{p}=2 \times 10-4)$, protein localization to nucleus and nuclear import $(p=5 \times 10-4)$. 